ARTIGO ORIGINAL

ORIGINAL ARTICLE

\title{
Avaliação crítica de estudos de custo-efetividade de medicamentos oncológicos recomendados para incorporação pela Conitec no Brasil
}

\author{
Critical appraisal of cost-effectiveness studies of oncologic drugs \\ recommended for incorporation by the Conitec in Brazil
}

Tatiane Bomfim Ribeiro', Moacyr R. Cuce Nobre',2, Antônio Carlos Coelho Campino³

DOI: $10.21115 / J B E S . v 12 . n 2 . p 155-63$

\section{Palavras-chave:}

custo-efetividade, setor público, oncologia, economia e organizações de saúde, economia da saúde

\section{Keywords:}

cost-effectiveness, public sector, oncology, health care economics and organizations, health economics

\section{RESUMO}

Objetivo: Realizar uma análise descritiva e avaliar a qualidade metodológica das análises de custo-efetividade dos medicamentos recomendados para incorporação pela Comissão Nacional de Incorporação de Tecnologias no SUS (Conitec) para o tratamento de câncer. Métodos: Um levantamento no site da Conitec foi realizado para identificar os relatórios de recomendação para medicamentos utilizados no tratamento de câncer, datados de janeiro/2012 a junho/2019. Os relatórios dos medicamentos com recomendação para incorporação e que apresentavam estudos de avaliação econômica em saúde foram incluídos. Uma análise descritiva e da qualidade metodológica (ferramenta Consensus on Health Economic Criteria [CHEC]) foi realizada. Resultados: Dez indicações diferentes, de oito medicamentos, para tratamento de câncer foram recomendadas pela Conitec de janeiro/2012 a junho/2019, porém somente cinco (50\%) dispunham de estudos de avaliação econômica. Um estudo foi excluído da avaliação da qualidade devido a limitações de acesso às informações. A qualidade metodológica foi variável nos estudos apresentados, e os domínios com pior avaliação se relacionavam a medida apropriada do desfecho e custos, discussões sobre ética, conflito de interesse e generalização dos dados. Observa-se uma falta de padronização na forma como foram realizadas as avaliações econômicas dos estudos submetidos à Conitec. Conclusão: Há grande heterogeneidade em relação à qualidade dos estudos de custo-efetividade de medicamentos oncológicos recomendados para incorporação pela Conitec e limitações metodológicas relevantes foram identificadas na maioria dos estudos.

\section{ABSTRACT}

Objective: To perform a descriptive analysis and evaluate the methodological quality of the costeffectiveness studies that based the "Comissão Nacional de Incorporação de Tecnologias no SUS" (Conitec) recommendations for cancer treatment. Methods: A survey on the Conitec website was performed in order to identify the recommendation reports for anticancer drugs, from January/2012 to June/2019. Reports for new drug incorporation that presented cost-effectiveness were included and analyzed. The methodological quality of these reports was assessed with the tool Consensus on Health Economic Criteria (CHEC). Results: Ten different indications of eight anticancer drugs were recommended by Conitec from 2012 to June/2019, but only five (50\%) had health economic analysis. One study was excluded from quality assessment due to limited information access. Methodological quality varies among the studies. The worst domains assessed were related to appropriate measurement of outcome and costs, ethics-related discussions, conflict of interest, and generalizability of the results. A lack of standardization was observed in Conitec's assessment on health economic studies submitted. Conclusion: There is a great heterogeneity in cost-effectiveness study quality of cancer drugs recommended by Conitec and relevant methodological limitations was noticed.

Recebido em: 15/05/2020. Aprovado para publicação em: 16/07/2020

1. Departamento de Medicina Preventiva, Faculdade de Medicina, Universidade de São Paulo, São Paulo, SP, Brasil.

2. Epidemiologia Clínica do Instituto do Coração (InCor) do Hospital das Clínicas da Universidade de São Paulo, São Paulo, SP, Brasil.

3. Departamento de Economia da Faculdade de Economia, Administração e Contabilidade da Universidade de São Paulo, São

Paulo, SP, Brasil.

Financiamento: Este estudo não recebeu qualquer tipo de financiamento.

Conflito de interesses: Os autores declaram que não há nenhum conflito de interesse para a realização deste trabalho.

Autor correspondente: Tatiane Bomfim Ribeiro. Faculdade de Medicina da Universidade de São Paulo. Av. Dr. Arnaldo, 455, Cerqueira César, São Paulo, SP, Brasil. CEP: 01246-903.E-mail: tatianeribeiro6@gmail.com 


\section{Introdução}

O câncer é a segunda maior causa de óbito em todo o mundo, sendo responsável por 9,5 milhões de mortes no último ano (Bray et al., 2018). Em 2018, havia 18,1 milhões de novos casos de câncer (Bray et al., 2018), caracterizando um problema de saúde pública (Bray et al., 2018). Segundo dados do Instituto Nacional do Câncer (Inca) de 2016, os cânceres com maior incidência no Brasil eram respectivamente: mama, próstata, pulmão/brônquios e colorretal (Inca, 2016), acompanhando a estatística mundial (Bray et al., 2018). A American Cancer Society, em 2015, relatou que a média do custo direto das despesas médicas com câncer foi de US\$ 80,2 bilhões (American Cancer Society, 2017), e uma perda de produtividade de US\$ 46,3 bilhões foi estimada devido à morte prematura por doenças oncológicas nos países do BRIC (Brasil, Rússia, China, África do Sul e Índia) (Pearce et al., 2018). No Brasil, o maior responsável pela perda de produtividade foi a neoplasia de pulmão (Pearce et al., 2018).

No contexto de grandes gastos e entrada no mercado de muitos novos produtos para a saúde, sobretudo em doenças com necessidades médicas não atendidas, como as oncológicas, as agências de avaliação de tecnologias em saúde (ATS) são importantes na determinação da eficiência, efetividade e segurança para subsidiar as recomendações de cobertura de novas tecnologias nos sistemas de saúde (Neumann et al., 2010). O National Institute for Health and Care Excellence (NICE), a agência britânica de ATS, desde 1999 avalia as tecnologias para o National Health Service (NHS), sistema de saúde nacional britânico. A inclusão de estudos econômicos é obrigatória e a padronização da medida é o QALY (sigla em inglês para anos de vida ajustados pela qualidade); Lakdawalla et al., 2018). Além disso, desde 2016, o NICE dispõe de um fundo específico para financiar os medicamentos contra o câncer, o Cancer Drug Fund (CDF), cujo objetivo é que o acesso ocorra de forma acelerada, particularmente para os medicamentos potencialmente inovadores, nos quais as evidências clínicas sobre os benefícios e a relação custo-efetividade não são suficientes para que o NHS realize a incorporação indiscriminadamente. Dessa forma, a aprovação se dá por um tempo determinado (geralmente em torno de dois anos), enquanto evidências adicionais são geradas por meio de dados coletados prospectivamente (NICE, 2020).

No Brasil, desde 2011, a Comissão Nacional de Avaliação de Tecnologias (Conitec) é responsável pela avaliação das tecnologias a serem disponibilizadas no Sistema Único de Saúde (SUS) (Brasil, 2011). No site da Conitec há todas as informações dos relatórios e recomendações, lembrando que a decisão final de incorporação é de responsabilidade do secretário da Secretaria de Ciência, Tecnologia e Insumos Estratégicos do Ministério da Saúde (SCTIE/MS) (Yuba et al., 2018). O demandante pode ser qualquer parte interessada na incorporação de uma nova tecnologia: Sociedade de Especialidade, Indústria Farmacêutica, Secretarias e outras instâncias governamentais (Brasil, 2011). Este submete à Conitec um documento com revisão sistemática e estudos de avaliação econômica em saúde (AES), como análises de custo-efetividade e impacto orçamentário. A AES tem como objetivo avaliar se uma intervenção é eficiente para a utilização dos recursos da sociedade (Conitec, 2019). Um estudo sugere que existe uma flexibilização da obrigatoriedade de AES para demandantes internos, ou seja, do próprio governo (Yuba et al., 2018).

Diversos instrumentos são utilizadas para avaliar a qualidade metodológica de AES, porém não há um consenso em relação à ferramenta de escolha, e um overview de revisões sistemáticas de estudos econômicos mostrou heterogeneidade nessas avaliações (Luhnen et al., 2019) e foram citados diferentes instrumentos: Drummond checklist (Drummond \& Jefferson, 1996), Philips checklist (Philips et al., 2004), CHEC-list (Evers et al., 2005) e QHES (Chiou et al., 2003).

Atualmente, não há publicações científicas acerca da qualidade dos estudos econômicos submetidos em dossiês de ATS no Brasil, dessa forma, o objetivo deste artigo é avaliar a qualidade metodológica e realizar uma análise descritiva das análises de custo-efetividade dos medicamentos oncológicos recomendados para incorporação pela Conitec.

\section{Métodos}

O site da Conitec (www.conitec.gov.br) foi consultado em 27 de junho de 2019 e uma busca manual foi realizada para a identificação dos medicamentos oncológicos que a Conitec recomendou para incorporação no SUS. Os critérios de elegibilidade incluíram terapias farmacológicas para tratamento de qualquer neoplasia; procedimentos ou exames diagnósticos foram excluídos. Os dados dos medicamentos para câncer recomendados pela Conitec foram extraídos do parecer de aprovação disponível no website; esses dados incluíram nome genérico, indicação, data recomendação da Conitec, demandante e estudo de AES (Sim ou Não; Desenho).

Os medicamentos que possuíam estudos de AES foram selecionados como objeto do presente estudo. A Lei de Acesso à Informação é um canal para solicitação de informações públicas, por meio do Sistema de Acesso à Informação: https://esic.cgu.gov.br. Um chamado foi aberto para solicitação dos documentos com estudos de avaliação econômica completos submetidos à Conitec pelo demandante. No dia 22 de julho a Conitec respondeu com a disponibilização dos mesmos relatórios que estavam disponíveis no site. Logo, esses documentos, publicados on-line pela Conitec, foram utilizados como objeto de avaliação.

A extração de dados incluiu informações referentes ao nome genérico do medicamento, ano de recomendação da Conitec, data da decisão, nome do demandante, classificação do tipo de demandante (interno, se órgão governamental ou 
externo, se outro), tipo de avaliação econômica realizada, intervenção e comparador do estudo, técnica de modelagem utilizada, horizonte temporal, taxa de desconto, desfechos clínicos incrementais e razão de custo-efetividade incremental (RCEI).

A avaliação da qualidade metodológica dos estudos econômicos foi realizada com os dados disponíveis nos documentos, por meio da ferramenta Consensus on Health Economic Criteria (CHEC-list) (Evers et al., 2005). O CHEC-list é um instrumento que dispõe de 19 perguntas; uma tradução não oficial para o português brasileiro foi realizada para fins de apresentação gráfica (Material Suplementar); as perguntas foram respondidas como "sim" para conforme e "não" para não conforme. Adicionalmente, uma avaliação da proporção média de questões com a resposta "sim" permitiu a classificação dos estudos, os quais foram considerados de alta qualidade se o escore estivesse acima de $75 \%$, de média qualidade se entre $51 \%$ e $74 \%$, e de baixa qualidade se abaixo de 50\% (Malet-Larrea et al., 2016)

\section{Resultados}

Desde o início de 2012, com a criação da Conitec, até o dia 21 de junho de 2019, oito medicamentos oncológicos foram recomendados para incorporação pela Conitec para tratamento do câncer, incluindo 10 indicações diferentes (trastuzumabe foi incorporado para três indicações) (Tabela 1). Somente cinco indicações incluíram estudos de custo-efetividade no relatório final da Conitec, tendo sido todos incluídos na análise descritiva, e o relatório do pertuzumabe incluiu as informações de dois demandantes diferentes (Tabela 2).

O documento submetido à Conitec para o pertuzumabe foi uma demanda mista da Sociedade Brasileira de Oncologia Clínica (SBOC) e do fabricante Roche. O relatório disponibilizado pela Conitec incluiu apenas a avaliação crítica, sem grandes detalhes dos estudos originais submetidos pelos demandantes, dessa forma, a avaliação da qualidade metodológica não foi realizada e o pertuzumabe foi incluído apenas

Tabela 1. Medicamentos recomendados pela Conitec para tratamento de câncer (janeiro/2012-junho/2019) e disponibilidade de estudo de custo-efetividade

\begin{tabular}{|c|c|c|c|c|}
\hline Nome genérico & Indicação & $\begin{array}{l}\text { Data decisão } \\
\text { Conitec }\end{array}$ & $\begin{array}{l}\text { Nome do demandante (interno ou } \\
\text { externo) }\end{array}$ & $\begin{array}{l}\text { Análise econômica } \\
\text { em saúde (custo- } \\
\text {-efetividade) }\end{array}$ \\
\hline Trastuzumabe & Câncer de mama avançado & Jul/2012 & $\begin{array}{l}\text { Secretaria de Atenção à Saúde (SAS) } \\
\text { do Ministério da Saúde (Interno) }\end{array}$ & Não \\
\hline Trastuzumabe & Câncer de mama em estágio inicial & $\mathrm{Jul} / 2012$ & Roche (externo) & Não \\
\hline Rituximabe & $\begin{array}{l}\text { Linfoma não Hodgkin de células b, } \\
\text { folicular, CD20 positivo, em 1a e 2a } \\
\text { linha }\end{array}$ & Set/2012 & $\begin{array}{l}\text { Departamento de Articulação de } \\
\text { Redes de Atenção à Saúde/Secretaria } \\
\text { de Atenção à Saúde - SAS/MS } \\
\text { (interno) }\end{array}$ & Não \\
\hline Gefitinibe & $\begin{array}{l}\text { Câncer de pulmão de células não } \\
\text { pequenas avançado ou metastático } \\
\text { mutação EGFR em } 1^{\text {a linha de }} \\
\text { tratamento }\end{array}$ & Nov/2013 & AstraZeneca (externo) & $\begin{array}{l}\text { Sim } \\
\text { Estudo do demandante }\end{array}$ \\
\hline Erlotinibe & $\begin{array}{l}\text { Câncer de pulmão de células não } \\
\text { pequenas avançado ou metastático } \\
\text { mutação EGFR em } 1^{\text {a }} \text { linha de } \\
\text { tratamento }\end{array}$ & Nov/2013 & Roche (externo) & $\begin{array}{l}\text { Sim } \\
\text { Estudo do demandante }\end{array}$ \\
\hline Imatinibe & $\begin{array}{l}\text { Adjuvante do tumor do estroma } \\
\text { gastrointestinal }\end{array}$ & $\mathrm{Jul} / 2014$ & $\begin{array}{l}\text { Secretaria de Atenção à Saúde - SAS/ } \\
\text { MS (interno) }\end{array}$ & Não \\
\hline Talidomida & Síndrome mielodisplásica & Dez/2014 & $\begin{array}{l}\text { Departamento de Gestão e } \\
\text { Incorporação de Tecnologias em } \\
\text { Saúde - DGITS/SCTIE } \\
\text { (interno) }\end{array}$ & Não \\
\hline Trastuzumabe & $\begin{array}{l}\text { Câncer de mama HER2-positivo } \\
\text { metastático em } 1 \text { a linha de } \\
\text { tratamento }\end{array}$ & Ago/2017 & $\begin{array}{l}\text { Secretaria de Ciência, Tecnologia e } \\
\text { Insumos Estratégicos (SCTIE/MS) } \\
\text { (interno) }\end{array}$ & $\begin{array}{l}\text { Sim } \\
\text { Estudo do demandante }\end{array}$ \\
\hline Pertuzumabe & $\begin{array}{l}\text { Câncer de mama HER2-positivo } \\
\text { metastático em } 1 \text { a linha de } \\
\text { tratamento }\end{array}$ & Dez/2017 & $\begin{array}{l}\text { Roche e Sociedade Brasileira de } \\
\text { Oncologia Clínica (externo) }\end{array}$ & $\begin{array}{l}\text { Sim } \\
\text { Avaliação da Conitec }\end{array}$ \\
\hline $\begin{array}{l}\text { Sunitinibe ou } \\
\text { pazopanibe }\end{array}$ & $\begin{array}{l}\text { Carcinoma renal de células claras } \\
\text { metastático em pacientes sem } \\
\text { tratamento prévio }\end{array}$ & Dez/2018 & $\begin{array}{l}\text { Sociedade Brasileira de Oncologia } \\
\text { Clínica (SBOC) (externo) }\end{array}$ & $\begin{array}{l}\text { Sim } \\
\text { Avaliação da Conitec }\end{array}$ \\
\hline
\end{tabular}


na análise descritiva. Nesse documento, a Conitec descreveu no relatório as limitações do modelo apresentado para o pertuzumabe. No documento da SBOC, foram relatadas inconsistências entre apresentação de dados e referência e dados de custos incompletos. O estudo submetido pela Roche foi amplamente criticado devido à utilização de comparações indiretas, além da escolha de horizonte temporal de 50 anos, o qual enviesa os resultados.

A Tabela 2 traz as informações dos estudos de análise de custo-efetividade recomendados pela Conitec. Todos os estudos utilizaram a técnica de Markov, e a avaliação do horizonte temporal variou entre 4 e 50 anos; a taxa de desconto utilizada foi de $3 \%$ ou 5\%. As RCEls apresentadas pelos estudos foram diversas: ano de vida livre de progressão, ano de vida ganho e ano de vida ajustado pela qualidade. Os valores de RCEI deste estudo variaram de $R \$ 26.631,51$ a R\$343.151,78, considerando que esses são valores concorrentes são avaliados por medidas diferentes (Tabela 3).

Na avaliação da qualidade dos estudos de análise de custo-efetividade presentes nos relatórios de recomendação da Conitec, identificou-se que a proporção de concordância com os critérios do CHEC para o gefitinibe, o erlotinibe, o sunitinibe/pazopanibe e o trastuzumabe foram, respectivamente, de 53\% (moderada), 32\% (baixa), 47\% (baixa) e 79\% (alta).

Na avaliação geral, metade dos domínios apresentou avaliação insatisfatória nas quatro análises de custo-efetividade incluídas; entre eles, podemos citar: desfechos medidos inapropriadamente, ausência de generalização dos dados discutida e ausência de discussão das questões éticas. Apenas um estudo completou os seguintes critérios: conflito de interesse descrito (pesquisadores e financiadores), conclusão concordante com o relato, custos futuros e desfechos descontados apropriadamente, desfechos relevantes identificados para cada alternativa, custos valorados apropriadamente e custos relevantes identificados para cada alternativa (Figura 1).

No presente estudo, todas as análises de custo-efetividade utilizaram dados publicados da literatura com diversos pressupostos, entre eles, tempo de transição entre os ciclos, valores estimados de progressão e mensuração de gastos com eventos adversos, probabilidades das medidas de efetividade etc. De modo geral, a maioria dos estudos não descreve em

Tabela 2. Descrição estudos de custo-efetividade de medicamentos recomendados pela Conitec para tratamento de câncer perspectiva do SUS

\begin{tabular}{|c|c|c|c|c|c|}
\hline $\begin{array}{l}\text { Medicamento, ano do } \\
\text { relatório da Conitec }\end{array}$ & $\begin{array}{l}\text { Grupo intervenção } \\
\text { vs. } \\
\text { Grupo controle }\end{array}$ & Modelo & $\begin{array}{l}\text { Descrição de } \\
\text { transições do modelo }\end{array}$ & $\begin{array}{l}\text { Horizonte temporal } \\
\text { Taxa de desconto }\end{array}$ & $\begin{array}{l}\text { CHEC Qualidade Global } \\
\text { (\% e proporção) }\end{array}$ \\
\hline \multirow[t]{2}{*}{ Gefitinibe, 2013} & $\begin{array}{l}\text { Gefitinibe } \\
\text { vs. }\end{array}$ & Markov & SLP - progressão - morte & 5 anos & Qualidade Moderada \\
\hline & $\begin{array}{l}\text { cis+dctx, cis+vin, cis+ } \\
\text { gen e carbo+ptx }\end{array}$ & & Ciclo de 21 dias & $5 \%$ & $53 \%(10 / 19)$ \\
\hline \multirow[t]{2}{*}{ Erlotinibe, 2013} & $\begin{array}{l}\text { Erlotinibe } \\
\text { vs. }\end{array}$ & Markov & SLP - progressão - morte & & Qualidade Baixa \\
\hline & cis+dctx e cis+ gen & & $\begin{array}{l}\text { Transição a qualquer } \\
\text { momento ciclos mensais }\end{array}$ & $5 \%$ & $32 \%(6 / 19)$ \\
\hline \multirow[t]{2}{*}{ Pertuzumabe, $2017^{a}$} & $\begin{array}{l}\text { Pertuzumabe + } \\
\text { trastuzumabe + QT }\end{array}$ & Markov & Progressão - morte & ND anos & Não se aplica ${ }^{a}$ \\
\hline & $\begin{array}{l}\text { VS. } \\
\text { QT }\end{array}$ & & Ciclos: ND & $3 \%$ & \\
\hline Pertuzumabe, $2017^{a}$ & $\begin{array}{l}\text { Pertuzumabe + } \\
\text { trastuzumabe + QT } \\
\text { vs. } \\
\text { QT }\end{array}$ & Markov & Dados modelo: ND & $\begin{array}{l}50 \text { anos e } 10 \text { anos } \\
\text { ND }\end{array}$ & Não se aplica ${ }^{a}$ \\
\hline Trastuzumabe, 2017 & $\begin{array}{l}\text { Trastuzumabe+dctx } \\
\text { vs. } \\
\text { dctx }\end{array}$ & Markov & $\begin{array}{l}\text { Doença estável - } \\
\text { progressão - morte } \\
\text { Ciclo mensal } \\
\text { (probabilidades) }\end{array}$ & $\begin{array}{l}4 \text { anos } \\
\text { ND }\end{array}$ & $\begin{array}{l}\text { Qualidade Alta } \\
\text { 79\% (15/19) }\end{array}$ \\
\hline \multirow[t]{2}{*}{$\begin{array}{l}\text { Sunitinibe ou } \\
\text { pazopanibe, } 2018^{b}\end{array}$} & $\begin{array}{l}\text { Sunitinibe ou } \\
\text { pazopanibe } \\
\text { vs. }\end{array}$ & Markov & $\begin{array}{l}\text { Progressão - suporte } \\
\text { clínico - morte }\end{array}$ & $\begin{array}{l}\text { Tempo de vida (anos } \\
\text { ND) }\end{array}$ & Qualidade Baixa \\
\hline & IFN-a & & $\begin{array}{l}\text { Ciclo de } 6 \text { semanas } \\
\text { (probabilidades) }\end{array}$ & $3 \%$ & $47 \%(9 / 19)^{b}$ \\
\hline
\end{tabular}

a Dados disponíveis são insuficientes para a avaliação integral da qualidade do estudo; dois demandantes compõem o mesmo relatório da Conitec. ${ }^{\text {b }}$ Documento na íntegra submetido pelo demandante não estava disponível. Avaliação da qualidade metodológica limitada ao relato da Conitec. Abreviaturas: carbo: carboplatina; cis: cisplatina; dctx: docetaxel; gen: gencitabina; IFN-a: interferon-alfa; ND: não descreve; ptx: paclitaxel; QT: quimioterapia; SLP: sobrevida livre de progressão; vin: vinorelbina. 
Tabela 3. Desfecho e razão de custo-efetividade incremental (RCEI) dos estudos de custo-efetividade de medicamentos recomendados pela Conitec para tratamento de câncer

\begin{tabular}{|c|c|c|}
\hline $\begin{array}{l}\text { Medicamento, ano do } \\
\text { relatório da Conitec }\end{array}$ & Desfecho incremental & Razão de custo-efetividade incremental (RCEI) \\
\hline Gefitinibe, 2013 & 0,327 ano de vida livre de progressão & Entre $R \$ 52.969$ e $R \$ 53.552 / a n o$ de vida livre de progressão \\
\hline Erlotinibe, 2013 & $\begin{array}{l}\text { 0,580 ano de vida ganho } \\
\text { 0,407 ano de vida ajustado por qualidade }\end{array}$ & $\begin{array}{l}\mathrm{R} \$ 26.631 / \mathrm{ano} \text { de vida ganho } \\
\mathrm{R} \$ 37.936 / \text { ano de vida ajustado por qualidade }\end{array}$ \\
\hline Pertuzumabe ${ }^{\mathrm{a}}, 2017$ & 15,7 meses de vida adicionais ${ }^{b}$ & $\mathrm{R} \$ 343.151 / a n o$ de vida extra \\
\hline Pertuzumabe ${ }^{a}, 2017$ & 15,7 meses de vida adicionais ${ }^{b}$ & $\begin{array}{l}\mathrm{R} \$ \text { 83.460/ano de vida salvo (50 anos) } \\
\mathrm{R} \$ 260.440 / \text { ano de vida extra ( } 10 \text { anos) }\end{array}$ \\
\hline Trastuzumabe, 2017 & 0,35 ano de vida ajustado por qualidade & $\mathrm{R} \$ 171.486 /$ ano de vida ajustado por qualidade \\
\hline Sunitinibe ou pazopanibec ${ }^{c}, 2018$ & 0,92 ano de vida ganho & $\begin{array}{l}\text { Sunitinibe - R\$ 105.836/ano de vida ganho } \\
\text { Pazopanibe - R\$ 65.219/ano de vida ganho }\end{array}$ \\
\hline
\end{tabular}

a Dados disponíveis são insuficientes para a avaliação integral da qualidade do estudo; dois demandantes compõem o mesmo relatório da Conitec. ${ }^{\mathrm{b}}$ Dados de desfecho encontrados na avaliação dos dados de efetividade das Evidências Científicas. ' Análise realizada pela Conitec; documento do demandante não se encontra disponível.
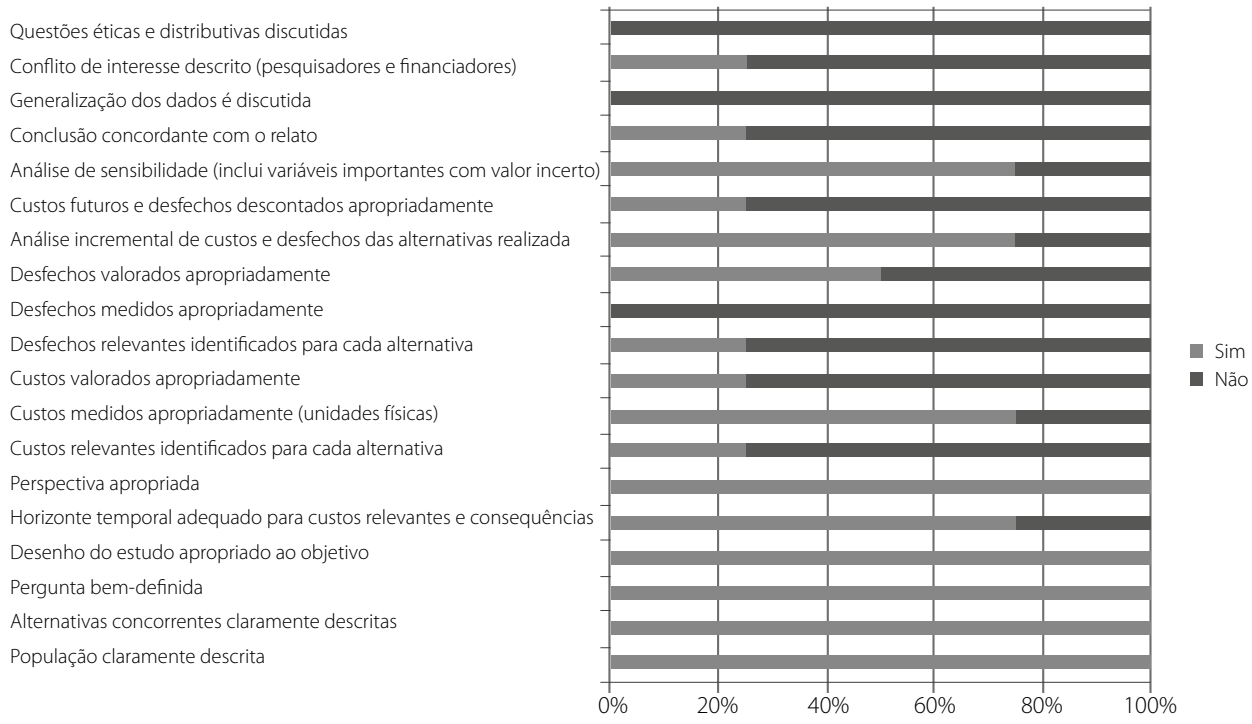

Figura 1. Avaliação da qualidade metodológica (instrumento CHEC - tradução não oficial) dos estudos de custo-efetividade de medicamentos oncológicos recomendados pela Conitec.

detalhes as probabilidades, etapas de transição e motivo da escolha do modelo. Nenhum dos estudos fez uma revisão completa da literatura para os dados de efetividade. Os dados de custos em geral eram em sua grande maioria descritos parcialmente, por exemplo, frequentemente os dados de eventos adversos não incluíam referências, ou não se detaIhou quais fatores corroboram aquela medida de efeito.

\section{Discussão}

O presente estudo mostrou que há heterogeneidade nos relatórios apresentados pela Conitec para subsidiar as recomendações de novos medicamentos oncológicos, e a maioria dos estudos de custo-efetividade possui limitações metodológicas, caracterizando incertezas.
Uma possível explicação para os dados encontrados neste estudo pode ser a dificuldade para o desenvolvimento de estudos de avaliação econômica no contexto brasileiro, no qual há muitas vezes carência de dados econômicos e clínicos, e possivelmente uma formação incipiente dos recursos humanos para gerar evidências de qualidade (Yuba et al., 2018), considerando a ATS ainda emergente no Brasil, com início em 2011 com a criação da Conitec.

Os modelos dos estudos de AES são construídos por meio de estimativas da realidade e diversos pressupostos são definidos, pois a maioria não utiliza dados de mundo real. No presente estudo, todas as análises de custo-efetividade utilizaram dados publicados da literatura, as quais muitas vezes não representam a realidade brasileira e do SUS. Outro princípio fundamental é a escolha do desfecho que deve ser 
de acordo com a medicina baseada em evidências. Os desfechos intermediários que são utilizados para predizer um efeito clínico significativo devem ser evitados (Rascati, 2013), sendo preferidos os resultados críticos para o paciente, como sobrevida e qualidade de vida.

Após a escolha do desfecho, a literatura sugere a elaboração de uma revisão sistemática para a seleção de estudos que avaliem a efetividade, e não apenas a eficácia. Nossos resultados mostraram que nenhum dos estudos fez essa avaliação completa da literatura previamente. O pressuposto de incluir dados de efetividade em um estudo de AES resultará, ao final, em um dado que pode ser chamado de "eficiente" (Palmer \& Torgerson, 1999).

Esse cenário de falhas metodológicas na condução das evidências apresentadas não se restringe ao Brasil. Um estudo publicado em 2009 avaliou os dados de efetividade e das análises de custo-efetividade da agência britânica, canadense e australiana, e fez críticas aos dados que incluíram limitações resultantes de desenho de estudo e uso de comparadores inadequados e desfechos intermediários não validados, porém, segundo os autores, as análises de custo-efetividade não apresentam grandes incertezas (Clement et al., 2009).

Um estudo publicado em 2018 avaliou as características de estudos econômicos em oncologia publicados no Brasil de 1998 a 2013. O câncer de mama foi o mais estudado e a maioria dos estudos econômicos completos era de análise de custo-efetividade. Nesse estudo de Campolina et al., a RCEI por QALY ganho estava entre USD 466.45 e USD 374,630.96, o que corresponderia a aproximadamente $\mathrm{R} \$ 2.100,00$ a $\mathrm{R} \$ 1,7 \mathrm{mi}-$ Ihão de reais (usando a taxa de câmbio de 1 USD $=R \$ 4,50$, de 04/03/2020) (Campolina et al., 2018). No nosso estudo, somente dois estudos utilizaram o QALY; a maioria utilizou o valor de anos de vida ganho, sem ajuste para a qualidade de vida.

A RCEl do nosso estudo variou de $\mathrm{R} \$ 26.631,51$ a $\mathrm{R} \$ 343.151,78$, valores concorrentes avaliados por medidas diferentes. O estudo de Yuba et al. (2018) avaliou comparativamente a RCEI das tecnologias aprovadas pela Conitec. Nessa análise, o gefitinibe foi a segunda tecnologia com maior RCEl, superando em duas vezes o limiar sugerido pela Organização Mundial da Saúde (OMS), de 1 a 3 PIB/per capita. A primeira tecnologia é o PET-CT para o câncer colorretal, com RCEl de R\$ 120.000,00/ano. O RCEl de aprovação do erlotinibe, incluído no presente estudo, foi o quinto maior entre todas as tecnologias com estudos econômicos aprovados pela Conitec (Brasil, 2011). O limiar de custo efetividade é uma grande discussão nas agências de ATS em todo o mundo. O NICE preconiza que todos os estudos econômicos devem incluir avaliação de custo-efetividade com apresentação dos valores em QALY; a disposição a pagar do Reino Unido foi definida como $£ 20,000 £$ e $£$ 30,000 por 7 anos QALYS. A justificativa para usar o QALY está no entendimento de que essa é a medida genérica que reflete mortalidade e também efeito na qualidade de vida (Briggs et al., 2006). No Brasil, ainda não existe um limiar de aceitabilidade ou uma medida-padrão, como o QALY (Soarez \& Novaes, 2017). Muitos estudos brasileiros utilizam o limiar de um relatório da OMS (2001), de 1 a 3 $\mathrm{PIB} /$ per capita, porém existe uma grande discussão acerca do tema, o que levou a uma reconsideração dessa recomendação pela OMS (Soarez \& Novaes, 2017).

Os nossos resultados mostraram uma grande heterogeneidade em relação à apresentação do relato, ao tipo de estudo de AES e à forma como a Conitec avaliou os dados submetidos pelos demandantes. Adicionalmente, apenas metade das recomendações para incorporação no SUS de medicamentos oncológicos tinha estudos econômicos, ou seja, existe uma incompatibilidade entre as evidências recomendadas e as efetivamente utilizadas, provavelmente havendo outros critérios utilizados na recomendação final da Conitec. No entanto, para a completa compreensão deste tema, seria importante analisar os fatores políticos e institucionais que afetam o uso de evidências no processo de tomada de decisão; na maioria dos casos, não se utilizam apenas dados técnicos e administrativos, mas também critérios políticos (Yuba et al., 2018).

A Conitec, comparada a outras agências de ATS, ainda é jovem, porém já mostra resultados positivos e sinaliza para aprimorar ainda mais o fluxo de incorporação de tecnologias no SUS. Recentemente, houve ações para aumentar a transparência das etapas e dos prazos estabelecidos para análise e a exitosa consolidação da participação da sociedade, por meio das consultas públicas. Apesar de a Diretriz Metodológica de Avaliação Econômica (iniciativa do SCTIE/ MS), publicada em 2014, se caracterizar como um importante recurso para que os demandantes orientem as análises (Brasil, 2014), nosso estudo mostrou que há grande heterogeneidade na forma de avaliação da Conitec para as AES na oncologia. Com o objetivo de aumentar a transparência e padronizar a forma de avaliação, sugerimos que a Conitec padronize a forma de avaliação dos estudos de AES. O NICE dispõe de um checklist próprio para avaliação crítica de estudos econômicos (NICE, 2019), dessa forma se padronizam a avaliação e o parecer técnico.

Nosso estudo ainda mostrou que apenas um estudo submetido por demandante interno apresentou alta qualidade metodológica. Os estudos submetidos por demandantes externos apresentaram qualidade moderada ou baixa. Devido à limitação de pequeno tamanho amostral (apenas cinco estudos incluídos na análise), não é possível fazer considerações sobre a qualidade por tipo de demandante ou evolução ao longo do tempo. Para isso, sugere-se que novos estudos sejam elaborados, incluindo diferentes patologias e eventualmente comparando a qualidade dos estudos para medicamentos incorporados vs. não incorporados. O presente estudo possui algumas limitações e, devido à impossibilidade de acesso ao estudo integral do demandante (mesmo após 
a tentativa de contato com a lei de acesso à informação), a avaliação completa da qualidade metodológica fica limitada, pois em alguns casos não estavam caracterizadas quais as informações adicionadas pelo corpo técnico da Conitec ou pelo próprio demandante.

\section{Conclusão}

A incorporação de novas tecnologias no SUS tem grande impacto no sistema de saúde, e a avaliação econômica completa é uma importante ferramenta para a recomendação de tecnologias eficientes que promovam a melhoria da sobrevida e da qualidade de vida dos indivíduos, associado à manutenção da integridade do sistema. A qualidade metodológica dos estudos de análise de custo-efetividade utilizados para recomendações da Conitec de novos medicamentos oncológicos foi variável e frequentemente apresentou limitações metodológicas. Os domínios com pior avaliação se relacionavam a medida apropriada do desfecho e custos, discussões sobre ética, conflito de interesses e generalização dos dados. Não há uma padronização na forma de avaliação e submissão dos estudos de custo-efetividade que compõem o dossiê de ATS, dessa forma, sugere-se que um padrão seja adotado pela Conitec para avaliar as demandas de estudos de AES.

\section{Agradecimentos}

Agradecemos a Tania Yuba, Elene Nardi e Barbara Oliveira pelos comentários no manuscrito.

\section{Referências bibliográficas}

American Cancer Society. Cancer Facts \& Figures 2017 [cited 25 February 2018]. Available from: https://www.cancer.org/research/cancer-factsstatistics/all-cancer-facts-figures/cancer-facts-figures-2017.html.

American Cancer Society. Economic Impact of Cancer [Internet]. Cancer.org. 2018 [cited 24 February 2018]. Available from: https://www.cancer.org/ cancer/cancer-basics/economic-impact-of-cancer.html.

Brasil. Lei no 12.401, de 28 de abril de 2011. Altera a Lei no 8.080, de 19 de setembro de 1990, para dispor sobre a assistência terapêutica e a incorporação de tecnologia em saúde no âmbito do Sistema Único de Saúde - SUS. Diário Oficial da União; 28 abr. 2011.

Brasil. Ministério da Saúde. Secretaria de Ciência, Tecnologia e Insumos Estratégicos. Departamento de Ciência e Tecnologia. Diretrizes metodológicas: estudos de avaliação econômica de tecnologias em saúde. 2a ed. Brasília: Ministério da Saúde; 2014.

Bray F, Ferlay J, Soerjomataram I, Siegel RL, Torre LA, Jemal A. Global cancer statistics 2018: GLOBOCAN estimates of incidence and mortality worldwide for 36 cancers in 185 countries. CA Cancer J Clin. 2018;68(6):394-424.

Briggs A, Sculpher M, Claxton K. Decision modelling for health economic evaluation. Oxford: Oxford University Press; 2006.

Campolina A, Yuba T, Decimoni T, Leandro R, Diz M, Novaes H, et al. Health Economic Evaluations of Cancer in Brazil: A Systematic Review. Front Publ Health. 2018;6:205.
Chiou CF, Hay JW, Wallace JF, Bloom BS, Neumann PJ, Sullivan SD, et al. Development and validation of a grading system for the quality of cost-effectiveness studies. Med Care.2003:41(1):32-44.

Clement F, Harris A, Li J, Yong K, Lee K, Manns B. Using Effectiveness and Cost-effectiveness to Make Drug Coverage Decisions: a comparison of Britain, Australia, and Canada. JAMA. 2009;302(13):1437-43.

Conitec. Recomendações da Conitec [Internet]. Conitec.gov.br. 2019 [cited 9 August 2019]. Available from: http://conitec.gov.br/decisoes-sobreincorporacoes.

Conitec. Recomendações da Conitec [Internet]. Erlotinib. Câncer de pulmão de células não-pequenas avançado ou metastático mutação EGFR Conitec.gov.br. 2019 [cited 9 August 2019]. Available from: http://conitec. gov.br/decisoes-sobre-incorporacoes.

Conitec. Recomendações da Conitec [Internet]. Gefitinib. Câncer de pulmão de células não pequenas avançado ou metastático mutação EGFR. Conitec.gov.br. 2019 [cited 9 August 2019]. Available from: http://conitec. gov.br/decisoes-sobre-incorporacoes.

Conitec. Recomendações da Conitec [Internet]. Imatinibe. Adjuvante do tumor do estroma gastrointestinal. Conitec.gov.br. 2019 [cited 9 August 2019]. Available from: http://conitec.gov.br/decisoes-sobreincorporacoes.

Conitec. Recomendações da Conitec [Internet]. Pertuzumab. Câncer de mama HER2-positivo metastático em primeira linha de tratamento associado ao trastuzumabe e docetaxel. Conitec.gov.br. 2019 [cited 9 August 2019]. Available from: http://conitec.gov.br/decisoes-sobreincorporacoes.

Conitec. Recomendações da Conitec [Internet]. Rituximab. Linfoma não Hodgkin de células b, folicular, CD20 positivo, em 1a e 2a linha. Conitec. gov.br. 2019 [cited 9 August 2019]. Available from: http://conitec.gov.br/ decisoes-sobre-incorporacoes.

Conitec. Recomendações da Conitec [Internet]. Sunitinibe ou pazopanib. Carcinoma renal de células claras metastático. Conitec.gov.br. 2019 [cited 9 August 2019]. Available from: http://conitec.gov.br/decisoessobre-incorporacoes.

Conitec. Recomendações da Conitec [Internet]. Talidomida. Síndrome Mielodisplásica. Conitec.gov.br. 2019 [cited 9 August 2019]. Available from: http://conitec.gov.br/decisoes-sobre-incorporacoes.

Conitec. Recomendações da Conitec [Internet]. Trastuzumab. Câncer de mama HER2-positivo metastático em primeira linha de tratamento. Conitec.gov.br. 2019 [cited 9 August 2019]. Available from: http://conitec. gov.br/decisoes-sobre-incorporacoes.

Conitec. Recomendações da Conitec [Internet]. Trastuzumabe. Câncer de mama avançado. Conitec.gov.br. 2019 [cited 9 August 2019]. Available from: http://conitec.gov.br/decisoes-sobre-incorporacoes.

Conitec. Recomendações da Conitec [Internet]. Trastuzumabe. Câncer de mama inicial. Conitec.gov.br. 2019 [cited 9 August 2019]. Available from: http://conitec.gov.br/decisoes-sobre-incorporacoes.

Drummond MF, Jefferson TO. Guidelines for authors and peer reviewers of economic submissions to the BMJ. BMJ. 1996;313(7052):275-83.

Evers S, Goossens M, de Vet H, van Tulder M, Ament A. Criteria list for assessment of methodological quality of economic evaluations: consensus on health economic criteria. Int J Technol Assess Health Care. 2005;21(2):240-5.

Instituto Nacional de Câncer José Alencar Gomes da Silva. Coordenação de Prevenção e Vigilância. Estimativa 2018: incidência de câncer no Brasil. Rio de Janeiro: Inca; 2016.

Lakdawalla DN, Doshi JA, Garrison LP Jr, Phelps CE, Basu A, Danzon PM. Defining elements of value in health care - a health economics approach: an ISPOR Special Task Force report [3]. Value Health. 2018;21(2):131-9. 
Luhnen M, Prediger B, Neugebauer E, Mathes T. Systematic reviews of health economic evaluations: A structured analysis of characteristics and methods applied. Res Synt Methods. 2019;10(2):195-206.

Malet-Larrea A, García-Cárdenas V, Sáez-Benito L, Benrimoj SI, Calvo B, Goyenechea E. Cost-effectiveness of professional pharmacy services in community pharmacy: a systematic review. Expert Rev Pharmacoecon Outcomes Res. 2016;16(6):747-58.

Neumann PJ, Drummond MF, Jönsson B, Luce BR, Schwartz JS, Siebert U, et al.; International Working Group for HTA Advancement. Are Key Principles for improved health technology assessment supported and used by health technology assessment organizations? Int J Technol Assess Health Care. 2010;26(1):71-8.

NICE - National Institute for Health and Care Excellence [Internet]. Quality appraisal checklist - economic evaluations [cited 9 August 2019]. Available from: https://www.nice.org.uk/process/pmg4/chapter/ appendix-i-quality-appraisal-checklist-economic-evaluations\#notes-onthe-use-of-the-economic-evaluations-checklist.

NICE - National Institute for Health and Care Excellence [Internet]. Cancer drugs funds [cited 6 August 2020]. Available from: https://www. nice.org.uk/about/what-we-do/our-programmes/nice-guidance/ nicetechnology-appraisal-guidance/cancer-drugs-fund.
Palmer S, Torgerson D. Economics notes: Definitions of efficiency. BMJ. 1999;318(7191):1136-6.

Pearce A, Sharp L, Hanly P, Barchuk A, Bray F, de Camargo Cancela M, et al. Productivity losses due to premature mortality from cancer in Brazil, Russia, India, China, and South Africa (BRICS): A population-based comparison. Cancer Epidemiol. 2018;53:27-34.

Philips Z, Ginnelly L, Sculpher M, Claxton K, Golder S, Riemsma R, et al. Review of guidelines for good practice in decision-analytic modelling in health technology assessment. Health Technol Assess. 2004;8(36):iii-158.

Rascati K. Essentials of pharmacoeconomics. Philadelphia: Lippincott Williams \& Wilkins; 2013.

Soarez P, Novaes H. Limiares de custo-efetividade e o Sistema Único de Saúde. Cad Saúde Pública. 2017;33(4).

Yuba TY, Novaes HMD, de Soárez PC. Challenges to decision-making processes in the national HTA agency in Brazil: operational procedures, evidence use and recommendations. Health Res Policy Syst. 2018;16(1):40. 


\section{MATERIAL SUPLEMENTAR}

\section{Tradução não oficial do CHEC}

CHEC original
Is the study population clearly described?
Are competing alternatives clearly described?
Is a well-defined research question posed in
answerable form?
Is the economic study design appropriate to the
stated objective?
Is the chosen time horizon appropriate to
include relevant costs and consequences?
Is the actual perspective chosen appropriate?
Are all important and relevant costs for each
alternative identified?
Are all costs measured appropriately in physical
units?
Are costs valued appropriately?
Are all important and relevant outcomes for
each alternative identified?

\begin{tabular}{l} 
Are all outcomes measured appropriately? \\
\hline Are outcomes valued appropriately? \\
\hline Is an incremental analysis of costs and \\
outcomes of alternatives performed? \\
Are all future costs and outcomes discounted \\
appropriately? \\
Are all important variables, whose values are \\
uncertain, appropriately subjected to sensitivity \\
analysis?
\end{tabular}
analysis?

Do the conclusions follow from the data reported?

Does the study discuss the generalizability of the results to other settings and patient/client groups?

Does the article indicate that there is no potential conflict of interest of study researcher(s) and funder(s)?

Are ethical and distributional issues discussed appropriately?

\section{Tradução não oficial}

A população do estudo está claramente descrita?

\section{As alternativas concorrentes estão} claramente descritas?

Uma questão de pesquisa bem definida é colocada de forma esclarecedora?

O desenho do estudo econômico é apropriado para o objetivo declarado?

O horizonte de tempo escolhido é apropriado para incluir custos e consequências relevantes?

A perspectiva real escolhida é apropriada?

Todos os custos importantes e relevantes para cada alternativa são identificados?

Todos os custos são medidos apropriadamente em unidades físicas?

Os custos são avaliados apropriadamente?

Todos os resultados importantes e relevantes para cada alternativa são identificados?

Todos os desfechos são medidos apropriadamente?

Os resultados são valorizados apropriadamente?

É realizada uma análise incremental dos custos e dos resultados das alternativas?

Todos os custos e resultados futuros são descontados apropriadamente?

Todas as variáveis importantes, cujos valores são incertos, são apropriadamente submetidas à análise de sensibilidade?

As conclusões decorrem dos dados reportados?

O estudo discute a generalização dos resultados para outros ambientes e grupos de pacientes/clientes?

$\mathrm{O}$ artigo indica que não há potencial conflito de interesses de pesquisador(es) e financiador(es) do estudo?

São discutidas questões éticas e distributivas de forma apropriada?

\section{Resumo das questões}

População claramente descrita

Alternativas concorrentes claramente descritas

Pergunta bem-definida

Desenho do estudo apropriado ao objetivo

Horizonte temporal adequado para incluir custos relevantes e consequências

Perspectiva apropriada

Custos relevantes identificados para cada alternativa

Custos medidos apropriadamente (unidades físicas)

Custos valorados apropriadamente

Desfechos relevantes identificados para cada alternativa

Desfechos medidos apropriadamente

Desfechos valorados apropriadamente

Análise incremental de custos e desfechos das alternativas realizada

Custos futuros e desfechos descontados apropriadamente

Análise de sensibilidade (inclui variáveis importantes com valor incerto)

Conclusão concordante com os dados reportados

Generalização dos resultados é discutida

Conflito de interesse descrito

(pesquisadores e financiadores)

Questões éticas e distributivas discutidas 